\title{
INVESTIGACIONES
}

\section{El efecto migratorio en la asistencia escolar en Chile*}

\author{
The migratory effect on school attendance in Chile
}

\author{
Leidy Y. García ${ }^{a}$, Miguel Friz Carrillo ${ }^{b}$ \\ ${ }^{a}$ Facultad de Economía y Negocios, Universidad de Talca. \\ lgarcia@utalca.cl \\ ${ }^{b}$ Facultad de Educación, Universidad del Bío-Bío. \\ mfriz@ubiobio.cl
}

\begin{abstract}
La migración implica un importante desafío en materia de integración educativa de los niños y jóvenes extranjeros en el sistema educativo. Por ello, el objetivo de este artículo es estudiar los factores socioeconómicos del hogar que determinan la asistencia escolar en Chile, como factor esencial para la integración de los inmigrantes. Para ello, se aplica el método cuantitativo de estimación de la probabilidad de asistencia escolar. Los resultados indican que las variables que determinan la asistencia escolar son edad, nacionalidad, tipo de hogar, zona, nivel educativo del jefe de hogar y su condición laboral, condición de pobreza; entre otras. Esto evidencia que la probabilidad de asistencia de un niño extranjero de asistir es casi un $11 \%$ menos que la de un niño nativo. De esta manera, el Estado debería promover y garantizar la cobertura universal de la educación de los niños y jóvenes migrantes como paso fundamental para la integración.
\end{abstract}

Palabras claves: matrícula, inclusión, asistencia escolar, inmigrantes.

\begin{abstract}
Migration implies an important challenge in the educational integration of foreign children and youth in the education system. Therefore, the objective of this article is to study the socioeconomic factors of the home that determine school attendance in Chile, as an essential factor for the integration of immigrants. For this, the quantitative method of estimating the probability of school attendance is applied. The results indicate that the variables that determine school attendance are age, nationality, type of home, area, educational level of the head of household and their working condition, poverty condition, among others. Evidence that the probability of attendance of a foreign child to attend is almost $11 \%$ less than that of a native child. Thus, the State should promote and guarantee universal coverage of the education of migrant children and young people, as an fundamental step for integration.
\end{abstract}

Key words: enrollment, inclusion, school attendance, immigrants.

\footnotetext{
"Este artículo fue realizado con el financiamiento de los proyectos Fondecyt Regular 1170369 y 1180993 , de la Agencia Nacional de Investigación y Desarrollo de Chile".
} 


\section{INTRODUCCIÓN}

Chile es un país pequeño en términos poblacionales, pero es atractivo para los inmigrantes especialmente sudamericanos, porque ofrece oportunidades económicas y estabilidad política. Según los datos del Ministerio de Desarrollo Social (2018) el porcentaje de la población chilena nacida fuera del país pasó de ser un 1,4\% en 2011 al 4,4\% en 2017. Esto conlleva un importante desafío para el país en integración de los extranjeros. Al respecto, García y Lillo (2017) muestra que los extranjeros están relativamente integrados en el mundo laboral, pero no así en la participación social y en el sistema educativo. Específicamente, en dicho trabajo se encontró que la asistencia escolar infantil de niños extranjeros presenta tasas bajas. De esta manera, es preocupante que los niños, especialmente los de grupos vulnerables como lo son los inmigrantes, no asistan a la escuela, pues a su vez tienden a estar segregados, por lo tanto, tienen mayores riesgos de inasistencia y abandono escolar (Lawson y Lawson, 2013).

La asistencia escolar es fundamental porque en los primeros años de educación se adquieren los conocimientos y habilidades básicas (lectura, escritura, hábitos, entre otros) que contribuyen al desarrollo de las capacidades individuales y donde se cimientan las bases del capital humano (Heckman y Mosso, 2014). En otras palabras, el hecho de que los niños en edad escolar no estén matriculados en un establecimiento educativo implica un riesgo para el desarrollo profesional futuro de estos y su estabilidad económica en el largo plazo (Heckman, Stixrud y Urzua, 2006).

Por ello, el objetivo principal de este artículo es estudiar los determinantes de la asistencia escolar de niños extranjeros desde la perspectiva del hogar migrante. Cabe destacar que esta investigación se aborda desde los factores asociados al hogar y no a la escuela por la disponibilidad de información representativa a nivel nacional para Chile. Asimismo, Epstein y Sehldon (2002) señalan que hay reconocimiento de la literatura internacional de la influencia de las familias en la asistencia escolar; además de la importancia de los factores económicos en la deserción escolar (Entwisle, Alexander y Olson, 2005). Así, los resultados de este trabajo son un aporte al estudio de la integración escolar, considerando que la educación es fundamental para acabar con la pobreza intergeneracional de las poblaciones migrantes y garantizar la participación exitosa en la fuerza de trabajo (Heckman y Mosso, 2014; Heckman, Stixrud y Urzua, 2006; Rumberger y Lamb, 2003).

Los métodos utilizados se basan en estimaciones econométricas de la probabilidad de asistencia escolar y el efecto promedio de ser extranjero. Este tipo de modelo permite conocer las variables que afectan la probabilidad de estar matriculado en una institución educativa y permite controlar por características de los niños, de los jóvenes y sus hogares.

\section{CONTEXTO: INTEGRACIÓN Y EDUCACIÓN}

Chile es un país atractivo en el ámbito suramericano para los migrantes, esto implica, entre otros aspectos, un importante desafío de integración de los extranjeros al país. Considerando los datos de la encuesta de Caracterización Socioeconómica de Chile (CASEN) 2017, más del $12,7 \%$ de la población extrajera inmigrante en Chile son niños menores de 14 años, un $47,8 \%$ adultos entre 30 y 59 años y un $15,4 \%$ adultos mayores. El ingreso, las tasas 
de ocupación laboral y el acceso al sistema privado de salud muestran que los migrantes en Chile tienen buenas perspectivas laborales (Tabla 1). Es decir, la población migrante tiene mejores indicadores laborales que los nacionales, en contraposición a lo que algunos estudios señalan sobre otros países de la OCDE y Europa Continental (Schnepf, 2007). Lo anterior se podría explicar por los requisitos laborales que deben cumplir los inmigrantes para obtener los permisos de residencia en Chile; estos incluyen un contrato de trabajo y para su renovación se requiere acreditar el pago de todas las imposiciones de salud y pensiones (Circular 264645 del 04/12/2009 del Ministerio del Interior, Gobierno de Chile).

Tabla 1. Chile: Indicadores de integración de población migrante vs. nacional, 2015 y 2017

\begin{tabular}{|c|c|c|c|c|c|}
\hline \multirow{2}{*}{\multicolumn{2}{|c|}{ Indicador }} & \multicolumn{2}{|c|}{2015} & \multicolumn{2}{|c|}{2017} \\
\hline & & \multirow{2}{*}{\begin{tabular}{c|} 
Extranjeros \\
$76.70 \%$ \\
\end{tabular}} & \multirow{2}{*}{\begin{tabular}{|c|} 
Nacionales \\
$57.70 \%$ \\
\end{tabular}} & \multirow{2}{*}{\begin{tabular}{|c|} 
Extranjeros \\
$73.87 \%$ \\
\end{tabular}} & \multirow{2}{*}{$\begin{array}{c}\text { Nacionales } \\
53.75 \% \\
\end{array}$} \\
\hline \multirow{6}{*}{$\begin{array}{l}\text { Condiciones } \\
\text { laborales e } \\
\text { ingreso }\end{array}$} & Tasa de ocupación & & & & \\
\hline & $\begin{array}{l}\text { Ingreso promedio mensual } \\
\text { por hogar (autónomo) }\end{array}$ & $\$ 518,926$ & $\$ 292,848$ & $\$ 681,444$ & $\$ 507,606$ \\
\hline & $\begin{array}{l}\text { Porcentaje de hogares en } \\
\text { Quintil I }\end{array}$ & $10.40 \%$ & $20.30 \%$ & $11.42 \%$ & $21.77 \%$ \\
\hline & $\begin{array}{l}\text { Porcentaje de hogares en } \\
\text { Quintil V }\end{array}$ & $35.70 \%$ & $19.60 \%$ & $26.14 \%$ & $15.61 \%$ \\
\hline & Tasa de pobreza (ingreso) & $9.70 \%$ & $11.70 \%$ & $10.26 \%$ & $8.51 \%$ \\
\hline & \begin{tabular}{|l}
$\begin{array}{l}\text { Tasa de pobreza } \\
\text { (multidimensional) }\end{array}$ \\
\end{tabular} & $23.00 \%$ & $20.80 \%$ & $22.10 \%$ & $18.42 \%$ \\
\hline \multirow{3}{*}{$\begin{array}{l}\text { Formación } \\
\text { (educación) }\end{array}$} & $\begin{array}{l}\text { Porcentaje con estudios } \\
\text { universitarios }\end{array}$ & $27.20 \%$ & $17.80 \%$ & $38.8 \%$ & $21.51 \%$ \\
\hline & $\begin{array}{l}\text { Años promedio de } \\
\text { educación }\end{array}$ & 12.6 & 11 & 13.1 & 11.1 \\
\hline & Tasa de asistencia escolar & $85.80 \%$ & $91.60 \%$ & $74.73 \%$ & $82.55 \%$ \\
\hline \multirow[t]{2}{*}{ Vivienda } & $\begin{array}{l}\text { Tenencia de vivienda } \\
\text { propia }\end{array}$ & $20.90 \%$ & $64.90 \%$ & $18.32 \%$ & $63.98 \%$ \\
\hline & Tasa de hacinamiento & $21.50 \%$ & $6.70 \%$ & $25.65 \%$ & $9.41 \%$ \\
\hline Salud & $\begin{array}{l}\text { Tasa de afiliación al } \\
\text { sistema privado de salud }\end{array}$ & $17.60 \%$ & $15.00 \%$ & $17.25 \%$ & $14.21 \%$ \\
\hline \multirow[t]{2}{*}{$\begin{array}{l}\text { Cohesión } \\
\text { social }\end{array}$} & Tasa de discriminación & $28.40 \%$ & $14.70 \%$ & $31.75 \%$ & $14.47 \%$ \\
\hline & $\begin{array}{l}\text { Tasa de participación } \\
\text { social }\end{array}$ & $18.50 \%$ & $26.60 \%$ & $18.24 \%$ & $27.94 \%$ \\
\hline
\end{tabular}

Fuente. Elaboración de los autores a partir de datos de la Encuesta de Caracterización Socioeconómica Nacional (CASEN), 2015 y 2017.

Al estudiar los indicadores de integración socio-económica y educativa se puede afirmar que los inmigrantes están relativamente integrados. Los extranjeros tienen mayores tasas de participación laboral, ingresos y afiliación a sistemas privados de salud. Como se observa 
en la Tabla 1, el nivel de escolaridad es mayor en el grupo de inmigrantes por la selectividad de las visas, lo que es consistente con los hallazgos de Thomas (2012) para el caso de inmigrantes de raza negra en Estados Unidos y acorde con la teoría del capital humano que indica que mientras mayores son los niveles educativos más altos son los ingresos (Becker, 1975; Clark y Martorell, 2014). Además, la evidencia empírica emanada del estudio de Peet, Fink y Fawzi (2015) muestra que los retornos a la educación son altos en América Latina.

No obstante, la debilidad de la integración socio-económica se encuentra en la baja participación formal en organizaciones sociales que solo alcanza el 18,4\% en los extranjeros. Peor aún, cuando se analizan los resultados de la tasa de asistencia escolar (número de niños en edad escolar matriculados con respecto al total), se encuentra que es esta es más baja para los extranjeros $(74.73 \%)$ que para los nacionales $(82.55 \%)$. $\mathrm{Al}$ respecto, Lawson y Lawson (2013) indican que la asistencia escolar normalmente es más baja en poblaciones segregadas y Chile posee un sistema educativo segregado (Valenzuela, Bellei y de los Ríos, 2014), lo cual es preocupante para un país que busca la igualdad de oportunidades y garantiza el derecho a educarse. Más aun, una tasa de asistencia escolar baja implica un desafío de política pública migratoria por la vulnerabilidad al desarrollo profesional de los niños (Heckman et al. 2006).

Los datos muestran que los niños en condiciones de asistencia escolar se concentran entre los 6 y 18 años de edad, es decir, deberían asistir a enseñanza básica y media. Como se puede apreciar en la Figura 1, un 36,3\% de los niños pertenece al tramo de 6 a 13 años, mientras que un $27,3 \%$ pertenece al tramo 13 a 18 años de edad. Para el caso de la muestra nacional, destacan los tramos 6 a 13 años y 13 a 18 años con un 36,2\% y un $27,4 \%$ respectivamente. En la muestra extranjera, se destacan los tramos de 6 a 13 años con un $41,8 \%$ y el tramo 13 a 18 años con un $29,4 \%$. Una similitud entre ambos grupos es la concentración de niños en los tramos de 6 a 18 años. Sin embargo, una diferencia destacable es que en los tramos de menor edad ( 0 a 2 años y 2 a 4 años) la proporción de niños nacionales es mayor que la extranjera. En el tramo de 4 a 6 años las proporciones son casi idénticas.

Figura 1. Distribución de la muestra por edad: nacionales y extranjeros (porcentajes)

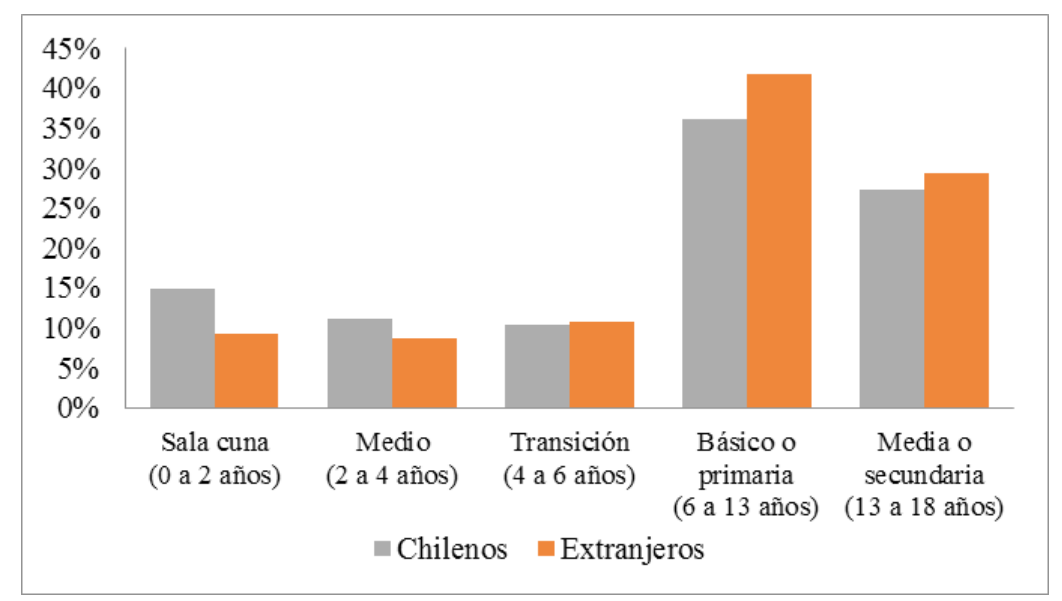


En la Figura 2 se puede apreciar que hay diferencias en las tasas de asistencia escolar por tramos de edad entre niños chilenos y extranjeros, a excepción de los infantes menores de dos años cuya tasa de asistencia a sala cuna es mayor para los extranjeros. Esto se explica por las menores redes de apoyo en la crianza inicial de los niños y la necesidad de cuidado infantil para que los padres puedan laborar. Esto es positivo porque la inscripción temprana ayuda a reducir las desigualdades en habilidades (Heckman, 2006; Magnuson, Lahaie y Waldfogel, 2006). Otra diferencia, más importante se presenta en la enseñanza media, en la que los nacionales tienen 6 puntos porcentuales más de asistencia que los inmigrantes.

Figura 2. Asistencia escolar por tramos de edad: nacionales y extranjeros

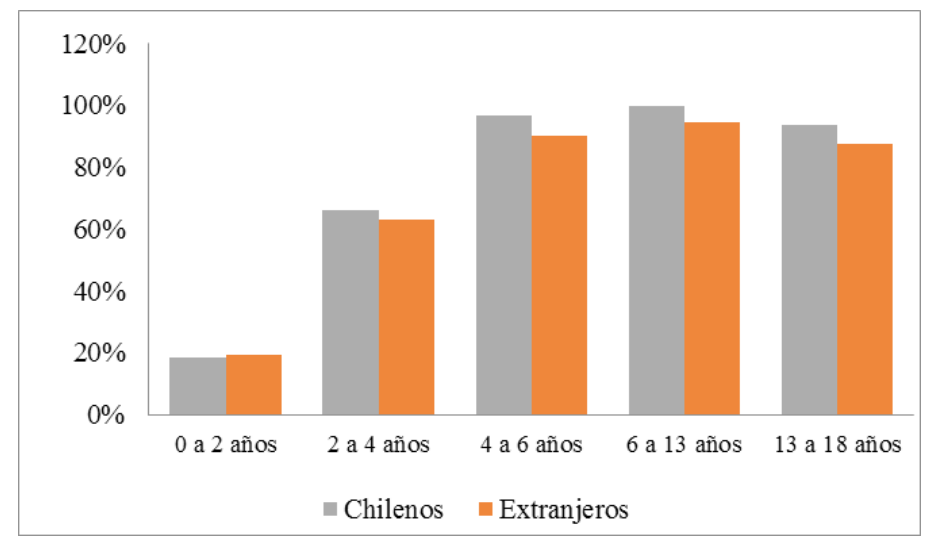

Considerando solo a los niños que asisten a instituciones de educación, se encontró que el $49,7 \%$ pertenecen a establecimientos particulares subvencionados, un $46,1 \%$ a municipales y un $4,2 \%$ a particulares pagados. Al comparar la tasa de matrícula por tipo de establecimiento y nacionalidad, se encuentra que hay más porcentaje de alumnos extranjeros matriculados en establecimientos municipales y particulares pagados $(49,1 \% \mathrm{y}$ $13,4 \%$, respectivamente), mientras que, para los nacionales, la mayor tasa de matrícula está en establecimientos particulares subvencionados $(49,8 \%)$. Lo anterior podría explicarse por los ingresos relativamente más altos que poseen los extranjeros en términos relativos (Tabla 1). De hecho, solo el 11,2\% de los hogares extranjeros pertenece al quintil I (los más pobres) y el 29,3\% al quintil V (los más ricos), mientras que para los nacionales estas proporciones son de $22,1 \%$ y $15,9 \%$, respectivamente. Es decir, se puede afirmar que el ingreso influencia el tipo de dependencia educacional que prefieren los padres para sus hijos.

\section{REVISIÓN DE LA LITERATURA}

El problema de la asistencia escolar infantil y el abandono ha sido estudiado en la literatura internacional desde diversos enfoques. De hecho, se considera que la participación escolar es de naturaleza multidimensional. Desde la teoría de la educación se considera que los factores que determinan principalmente la deserción están asociados al entorno que 
cubre la escuela y el aula (Bellei, 2013; Rumberger y Thomas, 2000) y las condiciones socioeconómicas del hogar (Alexander, Entwisle y Kabbani, 2001; Cairns, Cairns y Neckerman, 1989; Okumu, Nakajjo y Isoke, 2008). Además, estudios como el de Giorguli y Serratos (2009) señalan que las características y ambiente familiar son determinantes de los procesos de aprendizaje y las trayectorias escolares de los niños.

Desde la perspectiva económica, que estudia los impactos y efectos de la migración, es más relevante el punto de vista socioeconómico, así el problema de la asistencia escolar puede ser explorado desde las características propias del niño y el hogar al que pertenece (Okumu et al. 2008). Por lo tanto, este artículo se centra en los factores socioeconómicos del hogar que determinan la asistencia escolar. Cabe destacar que, siguiendo a McNabb (2018) y UNESCO (2012), se entiende por asistencia escolar al hecho de que los niños en edad escolar estén matriculados en la educación primaria.

Existe una amplia literatura que estudia la relación entre migración y asistencia escolar. Esta se enfoca principalmente en estudiar el efecto de los ingresos extras de los padres que migraron, mientras sus hijos se quedaron en su país de origen. Estos estudios señalan que hay un efecto positivo de la migración sobre la educación de los jóvenes, porque los ingresos vía remesas afectan la demanda de educación (Antman, 2008; Borraz, 2005), mientras que otros indican que los padres migrantes afectan negativamente la asistencia escolar porque genera un desincentivo a estudiar por las potenciales posibilidades de emigrar que tienen los hijos (Meza y Pederzini, 2009; McKenzie y Rapoport, 2006). Sin embargo, es poca la literatura internacional sobre la relación entre migración y asistencia escolar cuando se trata de familias migrantes, es decir, en la que tanto jefes de hogar como sus hijos migraron.

Sobre esta última, se encontró evidencia internacional de la preponderancia de los factores económicos en la deserción escolar. Estos estudios se basan en el análisis de las decisiones de trabajar o estudiar por parte de los niños y sus familias (Cabus y Witte, 2011; Paz y Cid, 2012; Rosenblum, Goldblatt y Moin, 2008; Sapelli y Torche, 2004). Sin embargo, en el contexto chileno se limita el análisis de la escolaridad desde ese enfoque de incentivos económicos vs. inversión en educación, porque el trabajo infantil es prohibido por la Ley $N^{\circ} 20.189$ y está prácticamente erradicado. Por ello, se hace más interesante estudiar la asistencia escolar de inmigrantes en Chile. Al respecto, trabajos como el de Rosenblum et al. (2008) muestran que factores socioeconómicos como el ingreso afecta la deserción escolar. Específicamente, ellos encuentran que los migrantes etíopes en Israel tienen mayores tasas de inasistencia mientras más bajo es el nivel socioeconómico del hogar.

Por un lado, Colding, Husted y Hummelgaard (2009) y Bratsberg, Raaum y Roed (2011) muestran que existen brechas importantes entre los niños nativos e inmigrantes en la asistencia escolar, de hecho, estos señalan que el abandono temprano es mucho mayor en los extranjeros y depende de la edad de migración. Por otro lado, hay estudios como el de Thomas (2012) que señalan que los migrantes de raza negra en Estados Unidos tienen ventajas y mejores resultados escolares que otros inmigrantes. No obstante, hay poca literatura que aborde la asistencia escolar considerando el hecho de que el alumno esté matriculado en una institución de educación en contexto migratorio. Por ejemplo, Sapelli y Torche (2002) aducen que las tasas de asistencia escolar dejan de ser un problema interesante de analizar por ser altas; pero este posee más importancia al observar las diferencias en la asistencia escolar entre migrantes y nacionales, más aun, en un sistema educativo que procura la cobertura universal en enseñanza básica y media. 


\section{METODOLOGÍA}

\subsection{DATOS Y ANÁLISIS DESCRIPTIVO}

Los datos fueron obtenidos de la Encuesta de Caracterización Socioeconómica (CASEN) del 2017, realizada por el Ministerio de Desarrollo Social de Chile. En esta se encuestó a 216.739 personas pertenecientes a 69.816 hogares. La población inmigrante en este país ascendía a 777.407 , lo que representó un 4.4\% de la población total en 2017. Del total de la población inmigrante, 159.084 individuos pertenecen a la categoría de niños, es decir, hasta los 18 años de edad lo que representa un $18,4 \%$ de la población extranjera y un 3,59\% de la población total de niños. Del total de niños y jóvenes, el $0,8 \%$ ya había terminado la enseñanza media.

La literatura considera como variables relevantes para explicar la escolaridad de un individuo la edad ${ }^{1}$, el origen (educación de los padres), la pobreza y el género (Holmes, 2003; Urzua, 2012). Por ello, las variables utilizadas para este estudio fueron edad, nacionalidad, tipo de hogar, género, zona, nivel educativo del jefe de hogar, condición de pobreza, condición laboral del jefe de hogar y número de hijos. Las estadísticas descriptivas de estas variables se presentan en la Tabla 2. Las características de la muestra de niños y jóvenes indican que, en promedio, viven en hogares biparentales, principalmente residen en zonas urbanas, en hogares con dos hijos, el jefe de hogar posee educación superior y se encuentra ocupado, lo que conlleva a que el hogar se encuentre en condiciones de no pobreza. Más aun, en términos generales, se puede apreciar diferencias significativas en todas las variables incluidas en el modelo entre extranjeros y nacionales con valores inferiores a 0,005 en todos los casos.

En general, las características de los escolares y sus hogares son similares en promedio, tanto para los nacionales como para los extranjeros (Tabla 2). Es decir, se podría asumir que hay homogeneidad en las variables de control entre grupos a excepción de la nacionalidad y el ingreso (condición de no pobreza); pero la diferencia más relevante se presenta en la asistencia escolar, especialmente en los niños en edad de asistir a la enseñanza media. Mientras que la diferencia entre los que asisten está en la tasa de matrícula en instituciones de educación particulares de los extranjeros en relación con los nacionales.

\subsubsection{Modelo de determinantes}

La metodología está basada en la literatura econométrica que ha aportado importantes avances para medir determinantes y efectos en problemas educacionales. En este artículo es de especial interés identificar las variables que determinan la probabilidad de asistencia escolar. Debido a que las estadísticas descriptivas muestran que los niños en edad escolar presentan similitudes en sus propias características y la de sus hogares, pero también diferencias en el ingreso y la tasa de asistencia escolar. Sin embargo, las estadísticas descriptivas no permiten saber la significancia de las variables. Por ello, se considera pertinente medir el efecto de ser extranjero sobre la probabilidad de asistencia escolar.

Para conocer los determinantes y estimar la probabilidad de dicha asistencia, se estiman modelos probit en los que la variable dependiente es la asistencia escolar de niños y jóvenes menores de 18 años de edad que viven en Chile. Esta es dicotómica:

Aslund et al. (2015) demuestran que la edad posee una relación causal en la integración de migrantes. 


\begin{tabular}{|c|c|c|c|c|c|c|c|c|c|c|}
\hline \multirow{3}{*}{ 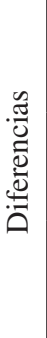 } & 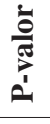 & $\begin{array}{l}8 \\
8 \\
0\end{array}$ & $\begin{array}{l}8 \\
8 \\
8\end{array}$ & $\begin{array}{l}8 \\
8 \\
ᄋ\end{array}$ & \begin{tabular}{l}
8 \\
\hdashline \\
0
\end{tabular} & $\begin{array}{l}8 \\
\stackrel{8}{\circ}\end{array}$ & $\begin{array}{l}8 \\
8 \\
ᄋ\end{array}$ & $\begin{array}{l}8 \\
8 \\
0\end{array}$ & \& & $\stackrel{8}{8}$ \\
\hline & $\vec{\theta}$ & $\vec{t}$ & 年 & $\sqrt{6}$ & $\stackrel{\vec{\infty}}{\dot{\infty}}$ & $\begin{array}{l}\circ \\
\stackrel{g}{q}\end{array}$ & $\begin{array}{l}\forall \\
\tilde{b} \\
i n\end{array}$ & $\stackrel{\infty}{\stackrel{\infty}{\curvearrowright}}$ & $\begin{array}{l}0 \\
0 \\
=\end{array}$ & di \\
\hline & $\ddot{\ddot{\theta}}$ & 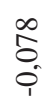 & $\begin{array}{l}\text { đ } \\
\text { ô } \\
1\end{array}$ & $\begin{array}{l}8 \\
8 \\
0 \\
1\end{array}$ & $\begin{array}{l}\mathbb{J} \\
0 \\
0\end{array}$ & $\begin{array}{l}\text { Oे } \\
\delta\end{array}$ & $\begin{array}{l}\stackrel{8}{ } \\
0 \\
\text { i }\end{array}$ & $\stackrel{n}{\tilde{0}}$ & $\begin{array}{l}2 \\
\infty \\
0 \\
0\end{array}$ & $\frac{n}{0}$ \\
\hline \multirow{5}{*}{ 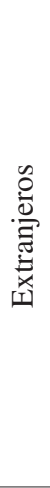 } & 莺 & - & $\stackrel{\infty}{-}$ & - & - & - & $\simeq$ & - & 0 & - \\
\hline & 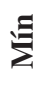 & 0 & 0 & - & 0 & 0 & 0 & 0 & 0 & 0 \\
\hline & $\vec{\theta}$ & 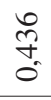 & 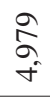 & $\begin{array}{l}\stackrel{0}{=} \\
=\end{array}$ & 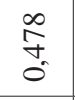 & $\frac{\overrightarrow{0}}{2}$ & $\stackrel{\substack{m \\
m}}{m}$ & $\underset{\dot{\theta}}{\vec{\nabla}}$ & $\begin{array}{l}\hat{2} \\
\stackrel{0}{0}\end{array}$ & $\begin{array}{l}\infty \\
\text { m. } \\
\text { m. }\end{array}$ \\
\hline & $\sum^{\stackrel{D}{e}}$ & - & $a$ & $\nabla$ & - & - & 0 & 0 & 0 & - \\
\hline & 晃 & $\frac{\text { J }}{\stackrel{5}{0}}$ & $\begin{array}{l}\text { 긍 } \\
\text { an }\end{array}$ & $\begin{array}{c}\underset{N}{\sim} \\
\text { ñ }\end{array}$ & $\begin{array}{l}\stackrel{\infty}{0} \\
\stackrel{0}{0}\end{array}$ & $\begin{array}{l}\overrightarrow{0} \\
\text { ᄋ̆ }\end{array}$ & $\begin{array}{l}\stackrel{8}{\circ} \\
\text { б. }\end{array}$ & $\begin{array}{l}\frac{\partial}{\tilde{\sigma}} \\
\text { }\end{array}$ & $\begin{array}{l}\text { Dे } \\
\text { ? }\end{array}$ & $\begin{array}{l}n \\
\infty \\
0\end{array}$ \\
\hline \multirow{5}{*}{$\begin{array}{l}\frac{\tilde{d}}{\tilde{J}} \\
\frac{\tilde{\sigma}}{\tilde{J}} \\
z\end{array}$} & 晃 & - & $\stackrel{\infty}{\sim}$ & $n$ & - & - & $\simeq$ & - & $\infty$ & - \\
\hline & 灵 & 0 & 0 & - & 0 & 0 & 0 & 0 & 0 & 0 \\
\hline & $\vec{\theta}$ & $\begin{array}{l}2 \\
\text { n. } \\
0\end{array}$ & $\begin{array}{l}\stackrel{2}{+} \\
\stackrel{\sim}{*}\end{array}$ & $\stackrel{\infty}{\stackrel{8}{-}}$ & $\stackrel{\infty}{\stackrel{\infty}{+}} \underset{0}{\circ}$ & 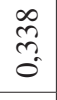 & $\begin{array}{l}\hat{\sigma} \\
\text { n. } \\
\text { n. }\end{array}$ & $\begin{array}{l}\hat{\infty} \\
\tilde{0} \\
\tilde{0}\end{array}$ & $\begin{array}{l}\text { है } \\
\text { ర్ } \\
0\end{array}$ & 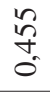 \\
\hline & 总 & - & $a$ & $\nabla$ & - & - & $n$ & 0 & 0 & - \\
\hline & 窇 & $\begin{array}{l}\text { त) } \\
\infty \\
0 \\
0\end{array}$ & $\underset{\text { নू }}{\stackrel{\Xi}{J}}$ & $\begin{array}{l}\tilde{N} \\
\tilde{n} \\
n\end{array}$ & $\begin{array}{l}\text { do } \\
0 \\
0\end{array}$ & $\begin{array}{l}\hat{O} \\
\infty \\
0 \\
0\end{array}$ & $\begin{array}{l}\text { ते } \\
\text { † }\end{array}$ & $\frac{+}{\infty}$ & $\begin{array}{l}\stackrel{\infty}{\sim} \\
\stackrel{0}{0}\end{array}$ & 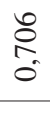 \\
\hline & 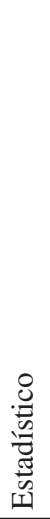 & 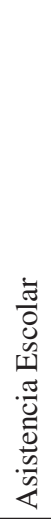 & 胥 & 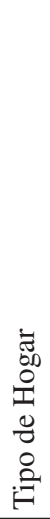 & 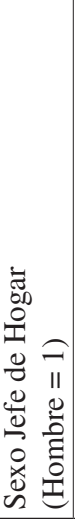 & 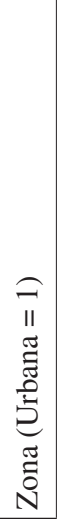 & 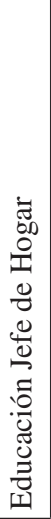 & 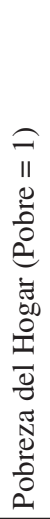 & 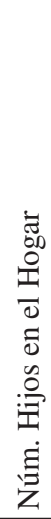 & 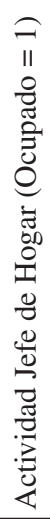 \\
\hline
\end{tabular}




$$
Y_{i}=\left\{\begin{array}{l}
1 \text { si asiste a una institución educativa } \\
0 \text { si no asiste }
\end{array}\right.
$$

Este tipo de modelos está basado en una función de probabilidad con distribución normal de la siguiente forma:

$$
\operatorname{Pr}\left(Y_{i}=1 / X_{i}\right)=\frac{1}{\sqrt{2 \pi}} \int_{-\infty}^{\beta^{\prime} X_{i}} e^{-t^{2} / 2} d t
$$

donde, $\beta$ es el vector de los coeficientes, $X$ representa la matriz de variables explicativas (edad, condición de extranjero, tipo de hogar, zona, educación del jefe de hogar, condición de pobreza, actividad, jefe de hogar y número de hijos) y $t$ a la variable normal estandarizada. Este se estima mediante el método de máxima verosimilitud.

\section{RESULTADOS Y DISCUSIÓN}

Los resultados del modelo de estimación de la probabilidad de asistencia escolar se presentan en la Tabla 3. Las medidas de bondad del ajuste muestran que el modelo efectivamente permite predecir la probabilidad de asistencia escolar. El porcentaje predicho correctamente supera el $91,1 \%$ de las observaciones de asistencia escolar y el Pseudo- $\mathrm{R}^{2}$ es de 0,33 . Las variables que son significativas y explican positivamente la asistencia escolar son la edad, ingresos sobre el umbral de no pobre, la educación, el número de hijos en el hogar y la actividad del jefe de hogar. Mientras que el tipo de hogar, pertenecer a una zona rural y ser extranjero; están asociadas negativamente a esta asistencia y son estadísticamente significativas.

Tabla 3. Estimación de la probabilidad de asistencia escolar: efectos marginales

\begin{tabular}{|l|c|c|c|c|c|c|}
\hline \multicolumn{1}{|c|}{ Asistencia Escolar } & Efecto marginal & $\mathrm{Dt}$ & $\mathrm{z}$ & \multicolumn{2}{c|}{$\mathrm{P}>\mathrm{z}$} & \multicolumn{2}{c|}{$\begin{array}{c}\text { Intervalo de } \\
\text { confianza (95\%) }\end{array}$} \\
\hline Extranjero & -0.101 & 0.001 & -113.680 & 0.000 & -0.103 & -0.099 \\
\hline Edad & 0.031 & 0.000 & 874.060 & 0.000 & 0.030 & 0.031 \\
\hline Tipo de Hogar* & 0.002 & 0.000 & 10.840 & 0.000 & 0.002 & 0.002 \\
\hline $\begin{array}{l}\text { Sexo Jefe de Hogar } \\
\text { (Hombre = 1) }\end{array}$ & -0.010 & 0.000 & -28.160 & 0.000 & -0.011 & -0.010 \\
\hline Zona (Urbana = 1) & 0.012 & 0.001 & 25.530 & 0.000 & 0.011 & 0.012 \\
\hline Educación Jefe de Hogar & 0.002 & 0.000 & 45.480 & 0.000 & 0.002 & 0.002 \\
\hline $\begin{array}{l}\text { Pobreza del Hogar } \\
\text { (Pobre = 1) }\end{array}$ & -0.051 & 0.000 & -132.180 & 0.000 & -0.052 & -0.050 \\
\hline \begin{tabular}{l} 
Núm. Hijos en el Hogar \\
\hline $\begin{array}{l}\text { Actividad Jefe de Hogar } \\
\text { (Ocupado= 1) }\end{array}$
\end{tabular}$-0.543$ & 0.003 & -175.760 & 0.000 & -0.549 & -0.537 \\
\hline
\end{tabular}




\begin{tabular}{|l|c|c|c|c|l|l|}
\hline Probabilidad Observada & 0.824 & & N & 3808014 & & \\
\hline Probabilidad Predicha & 0.917 & & LR chi $^{2}$ & $1.20 \mathrm{E}+06$ & & \\
\hline Prob > chi2 & 0.00 & & & & & \\
\hline Log likelihood & -1173251 & & Pseudo R $^{2}$ & 0.337 & & \\
\hline
\end{tabular}

Nota. * La variable toma los valores de 1: monoparental, 2: nuclear monoparental, 3: extendida monoparental, 4: nuclear biparental, 5: extendida biparental. ** La variable toma los valores de 1, inactivo, 2, desocupado, 3, ocupado. $D f / d x$ es el efecto marginal de la variable explicativa en la dependiente, $P>|z|$ es el valor $\mathrm{P}$ del test de estadístico, $D t$ es la desviación estándar. El modelo considera factores de expansión.

La edad es un factor determinante a la hora de medir la asistencia escolar. La variable es significativa al $100 \%$ y muestra un efecto marginal positivo. Esto es consistente con las costumbres y políticas de educación básica chilena en la que la mayoría de los niños inicia su asistencia escolar después de los cinco años. De hecho, solo desde el 2012 se ha promovido como política pública la asistencia a sala cunas y jardines infantiles como una forma de promover el desarrollo temprano de las habilidades (Pardo y Woodrow, 2014). Al respecto, los datos indican que la asistencia para los menores de 8 años es de un $65,72 \%$ mientras que para los mayores de 8 años asciende a un $96,75 \%$.

La probabilidad de asistencia escolar es más baja para los hogares de tamaño extendido. Lo anterior está justificado principalmente en que la variable toma un orden relacionado con la cantidad de adultos en el hogar. Es decir, a medida que existe una mayor cantidad de adultos, los niños asisten más tarde al colegio, lo que es evidente, porque los hogares monoparentales requieren del cuidado de los niños para que los jefes de hogar puedan trabajar. Así, los hogares con menos adultos llevan a los niños y jóvenes a las instituciones de educación para que sean cuidados, mientras que en hogares con más adultos el cuidado de estos se realiza en el hogar. A su vez, lo anterior es consistente con la otra variable asociada al tamaño del hogar, que es número de hijos en edad escolar. Además, se encuentra en la misma línea de los resultados encontrados por Sapelli y Torche (2004). Por tanto, en las familias más numerosas tiende a disminuir la probabilidad de asistencia escolar.

Un aspecto relevante del modelo es que muestra que efectivamente hay diferencia en la probabilidad de asistencia escolar entre niños extranjeros y chilenos, porque la variable dummy asociada a los inmigrantes es estadísticamente significativa a más de un $95 \%$ de nivel de confianza. Específicamente, los niños extranjeros tienen un 10,11\% menos de probabilidad de asistir a una institución educativa escolar (Tabla 3).

En cuanto a las variables socioeconómicas se encuentra que la educación del jefe de hogar y los ingresos que conduzcan a que el hogar sea considerado como "no pobre", son relevantes en la probabilidad de asistencia escolar. Lo que es consistente con los resultados de Holmes (2003). Los efectos de estas variables son positivos, es decir, a mayor grado de educación, los ingresos y la ocupación del jefe de hogar, mayor es la asistencia escolar de sus hijos. Además, están en la misma línea del efecto de la "cultura de la migración" de Giorguli y Serratos (2009) que señala que la prevalencia de la migración afecta negativamente la inscripción de los niños en los establecimientos de educación. 


\section{CONCLUSIONES}

La asistencia escolar es un pilar para educación y el desarrollo de los niños, sin embargo, la evidencia muestra que la tasa de matrícula escolar es más baja para los extranjeros $(74,3 \%$ equivalente a un $11 \%$ menos que la tasa de niños nacionales o nativos en 2017). Por lo tanto, es importante estudiar sus determinantes. Las estimaciones del modelo indican que los factores más relevantes que explican la asistencia provienen del origen del niño, la situación socioeconómica del hogar y sus características demográficas. Específicamente, las variables que son significativas y explican positivamente la asistencia escolar son la edad, ingresos sobre el umbral de no pobre, la educación y la actividad del jefe de hogar. Mientras que el tipo de hogar, pertenecer a una zona rural y ser extranjero están asociadas negativamente a la asistencia escolar y son estadísticamente significativas. De hecho, un niño extranjero tiene un 10,1\% menos de probabilidad de asistir a una institución escolar.

Por lo tanto, el Estado debería hacerse cargo de garantizar efectivamente la matrícula y asistencia escolar de los niños migrantes, especialmente en las zonas rurales y para hogares de bajos ingresos. Como señalan Chen y Feng (2013), el acceso a las escuelas es el factor clave que determina la calidad de la educación que reciben los niños migrantes. Más aún, las políticas migratorias deben reconocer los riesgos y desafíos que enfrentan los niños inmigrantes. Esta debe abordar las necesidades inmediatas y las perspectivas de largo plazo de los niños extranjeros y sus familias. Además, el acceso a la educación y el éxito en el egreso determinan las trayectorias futuras de desarrollo de capital humano del país (Heckman y Mosso, 2014). Así, constituye un desafío para Chile promover la cobertura universal de la educación básica y media de los migrantes.

El análisis realizado al problema de la asistencia escolar de niños extranjeros constituye una mirada interesante desde la perspectiva de la caracterización de los hogares. Sin embargo, se deberían realizar estudios a futuro que complementen la mirada de la asistencia escolar desde el hogar con la escuela y el entorno. Autores como Bellei (2013) señalan que la asistencia escolar puede ser determinada por el ambiente en el aula y la institución educativa, mientras que De Witte et al. (2013) indican que la asistencia escolar debe ser estudiada como multidimensional; para esto se requiere más información sobre los migrantes que aún no está disponible para el caso chileno.

\section{REFERENCIAS BIBLIOGRÁFICAS}

Alexander, K., Entwisle, D. y Kabbani, N. (2001). The dropout process in life course perspective: Early risk factors at home and school. Teachers college record, 103(5), 760-822. doi:10.1111/01614681.00134.

Antman, F. (2008). Parental Migration and Children's Education in Mexico: How Important is Child Age at the Time of Parent's Migration? Ponencia presentada en la Reunión Anual de la Population Association of America, Nueva Orleans, 17-19 de abril. Recuperado de http:// paa2008.princeton.edu/download.aspx?submissionId=80796.

Aslund, O., Böhlmark, A. y Skans, O. N. (2015). Childhood and family experiences and the social integration of young migrants. Labour Economics, (35), 135-144.

Becker, G. (1975). Human Capital and The Personal Distribution of Income: An Analytical Approach (W.S. Woytinsky Lecture). En G. Becker, Human Capital (2nd edition). New York: Columbia University Press. 
Bellei, C. (2013). El estudio de la segregación socioeconómica y académica de la educación chilena. Estudios Pedagógicos, 39(1), 209-229. doi: 10.4067/S0718-0705201300010001.

Borraz, F. (2005). Assessing the Impact of Remittances on Schooling: the Mexican Experience. Global Economy Journal, 5(1), 1-30. doi: 10.2202/1524-5861.1054.

Bratsberg, B., Raaum, O. y Roed, K., (2011). Educating children of immigrants: closing the gap in norwegian schools. IZA Discussion Paper, (6138), 1-36.

Cabus, S. y De Witte, K., (2011). Does school time matter? On the impact of compulsory education age on school dropout. Economics of Education Review, 30(6), 1384-1398.

Cairns, R., Cairns, B. y Neckerman, H. (1989). Early school dropout: Configurations and determinants. Child Development, 60(6), 1437-1452. doi: 10.2307/1130933.

Chen, Y. y Feng, S. (2013). Access to public schools and the education of migrant children in China. China Economic Review, 26(1), 75-88.

Clark, D. y Martorell, P. (2014). The signaling value of a high school diploma. Journal of Political Economy, 122(2), 282-318. doi: 10.1086/675238.

Colding, B., Husted, L. y Hummelgaard, H. (2009). Educational progression of second-generation immigrants and immigrant children. Economics of Education Review, 28(4), 434-443. doi: 10.1016/j.econedurev.2007.08.004.

De Witte, K., Cabus, S., Thyssen, G., Groot, W. y Brink, H. (2013). A critical review of the literature on school dropout. Educational Research Review, 10, 13-28. doi: 10.1016/j.edurev.2013.05.002.

Entwisle, D., Alexander, K. y Olson, L. (2005). First grade and educational attainment by age 22: a new story. American journal of sociology, 110(5), 1458-1502. doi: 10.1086/428444.

Epstein, J. y Sehldon, S. (2002). Present and accounted for: Improving student attendance through family and community involvement. Journal of Educational Research, 95(5), 308-318. doi: 10.1080/00220670209596604.

García, L. y Lillo, F. (2017). Integración de migrantes en Chile: una Mirada desde la Caracterización Socioeconómica de los Hogares. En Experiencias de Investigación en Ciencias Administrativas y Económicas en América Latina: una Perspectiva de Diferentes Proyectos (pp. 201-213). Quito: UIDE.

Giorguli, S. y Serratos, I. (2009). El impacto de la migración internacional sobre la asistencia escolar en México: ¿paradojas de la migración? En P. Leite y S, Giorguili, Las políticas públicas ante los retos de la migración mexicana a Estados Unidos (pp. 313-344). México, D.F.: Consejo Nacional de Población.

Heckman, J. (2006). Skill formation and the economics of investing in disadvantaged children. Science, 312(5782), 1900-1902. doi: 10.1126/science.1128898.

Heckman, J. y Mosso, S. (2014). The economics of human development and social mobility. Annual Review of Economics, 6(1), 689-733. doi: 10.1146/annurev-economics-080213-040753.

Heckman, J., Stixrud, J. y Urzua, S. (2006). The effects of cognitive and noncognitive abilities on labor market outcomes and social behavior. Journal of Labor Economics, 24(3), 411-482. doi: $10.1086 / 504455$.

Holmes, J. (2003). Measuring the determinants of school completion in Pakistan: Analysis of censoring and selection bias. Economics of Education Review, 22(3), 249-264. doi: 10.1016/ S0272-7757(02)00024-9.

Lawson, M. y Lawson, H. (2013). New conceptual frameworks for student engagement research, policy, and practice. Review of Educational Research, 83(3),432-479. doi: 10.3102/0034654313480891.

Magnuson, K., Lahaie, C. y Waldfogel, J. (2006). Preschool and school readiness of children of immigrant. Social Science Quarterly, 87(5), 1241-1262. doi: 10.1177/1476718X18761218.

Mckenzie, D. y Rapoport, H. (2006). Can migration reduce educational attainment? Evidence from Mexico. World Bank Policy Research Working Paper, 3952, 1-42.

McNabb, K. (2018). Exploring regional and gender disparities in Beninese primary school attendance: a multilevel approach. Education Economics, 26(5), 534-556. doi: 
10.1080/09645292.2018.1426732.

Meza, L. y Pederzini, C. (2009). Migración internacional y escolaridad como medios alternativos de movilidad social: el caso de México. Estudios Económicos (número extraordinario), 163-206.

Ministerio de Desarrollo Social. (2018). Resultados Casen 2017. Recuperado de http://observatorio. ministeriodesarrollosocial.gob.cl/casen-multidimensional/casen/docs/Resultados_Inmigran tes_casen_2017.pdf .

Ministerio del Interior, Gobierno de Chile. (2009, 4 de diciembre). Circular n ${ }^{\circ} 264645$, Santiago de Chile.

Okumu, I. M., Nakajjo, A. \& Isoke, D. (2008). Socio-economic determinants of primary school dropout: the logistical model analysis. Mpra Paper, 7851. Recuperado de: https://unevoc.unesco. org/e-forum/socioeco\%20determinants\%20ps\%20dropout\%202008.pdf.

Pardo, M, y Woodrow, C. (2014). Improving the quality of early childhood education in Chile: Tensions between public policy and teacher discourses over the schoolarisation of early childhood education. International Journal of Early Childhood, 46(1), 101-115. doi: 10.1007/ s13158-014-0102-0.

Paz, J. A. y Cid, J. C. (2012). Determinantes de la asistencia escolar de los jóvenes en la Argentina. Revista Electrónica de Investigación Educativa, 14(1), 136-152.

Peet, E., Fink, G. y Fawzi, W. (2015). Returns to education in developing countries. Evidence from the living standards and measurement study surveys. Economics of Education Review, 49, 6990. doi: 10.1016/j.econedurev.2015.08.002.

Rumberger, R. y Lamb, S. (2003). The early employment and further education experiences of high school dropouts: a comparative study of the United States and Australia. Economics of Education Review, 22(4), 353-366. doi: 10.1016/S0272-7757(02)00038-9.

Rumberger, R. W. y Thomas, S. L. (2000). Distribution of Dropout and Turnover Rates among Urban and Suburban High Schools. Sociology Education, 73(1), 39-67. doi: 10.2307/2673198.

Sapelli, C., y Torche, A. (2002). Subsidios al alumno o a la escuela: efectos sobre la elección de colegios públicos. Cuadernos de Economía, 39(117), 175-202. doi: 10.4067/S071768212002011700002.

Sapelli, C., y Torche, A. (2004). Deserción Escolar y Trabajo Juvenil: ¿Dos Caras de Una Misma Decisión? Cuadernos de Economía, 41(123), 173-198. doi: 10.4067/S0717-68212004012300001.

Schnepf, S. (2007). Immigrants' educational disadvantage: An examination across ten countries and three surveys. Journal of Population Economics, 20(3), 527-545. doi: 10.1007/s00148-0060102-y.

Thomas, K. (2012). Migration processes, familial characteristics, and schooling dropout among black youths. Demography, 49(2), 477-498. doi: 10.1007/s13524-012-0091-2.

Valenzuela, J., Bellei, C. y de los Ríos, D. (2014). Socioeconomic school segregation in a marketoriented educational system. The case of Chile. Journal of Education Policy, 29(2), 217-241. doi: 10.1080/02680939.2013.806995.

UNESCO (2012). UIS Frequently Asked Questions. Education Statistics. Recueperado de http:// www.uis.unesco.org/Education/Documents/FAQ-education-statistics-rev5-en.pdf. 
